\title{
Proton structure measurements and PDFs at HERA
}

\author{
Katerina Lipka for the $\mathrm{H} 1$ and ZEUS Collaborations \\ DESY, Notkestrasse 85, 22607 Hamburg, Germany
}

\begin{abstract}
Recent measurements of inclusive and semi-inclusive deep inelastic scattering in electron-proton collisions at HERA are reviewed. These measurements are used to determine the parton distribution functions (PDFs) of the proton, a necessary input to almost all theory predictions for hadron colliders. An introduction to the PDF determination with an emphasis on HERA PDFs is presented. Theory predictions based on HERAPDF are compared to a selection of recent LHC and Tevatron measurements. The impact of jet and charm production measurements in DIS at HERA on the PDFs is discussed.
\end{abstract}

Keywords: deep inelastic scattering, parton distribution functions, jets, charm, benchmarking

\section{Introduction}

A deep understanding of proton structure is one of the most important topics in modern particle physics. A precise knowledge of the parton distribution functions of the proton is essential in order to make predictions for the Standard Model and beyond the Standard Model processes at hadron colliders.

The cross sections of processes in proton-(anti)proton collisions are factorized by a convolution of the matrix element of parton-parton interaction and the proton structure. The latter is described by parton distribution functions (PDFs). A PDF, $f_{i}\left(x, Q^{2}\right)$, represents the probability of finding in the proton a parton $i$ (quark or gluon) carrying a fraction $x$ of the proton momentum, with $Q$ being the energy scale of the hard interaction. In case of proton-(anti)proton interaction PDFs of both hadrons enter multiplicatively into the calculation of the process cross section. Therefore the precision of the PDFs is of particular importance for accurate crosssection predictions. In the last decades, the measurements of lepton-nucleon and proton-antiproton scattering have been used to determine the proton PDFs.

The kinematic ranges accessible by the different experiments where PDFs can be determined are shown in Fig. 1. At low to medium $x$ the PDFs are constrained

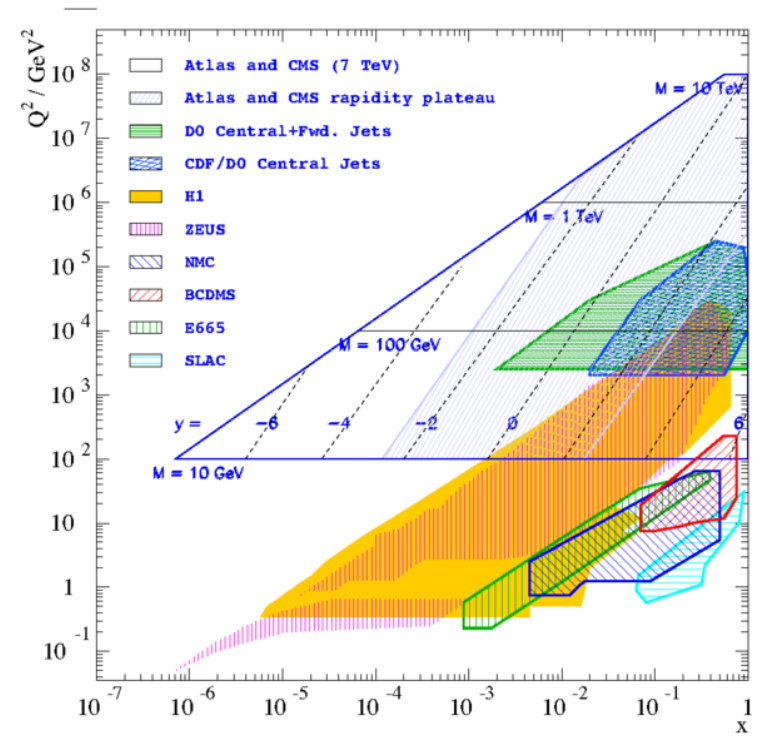

Figure 1: The ranges of $Q^{2}$ and $x$ accessible by fixed target experiments and in ep and proton-(anti)proton collisions.

by HERA data. The measurements at fixed target experiments and the Tevatron contribute mainly at high $x$. The recent precise data from the Tevatron and the LHC 
experiments have the potential to improve the precision on the PDFs further.

\section{Proton Structure and DIS at HERA}

The knowledge of the proton PDFs is obtained to a large extent from the measurements of the structure functions in deep inelastic scattering (DIS) experiments. In Fig. 2 the diagram for DIS is represented. The lepton

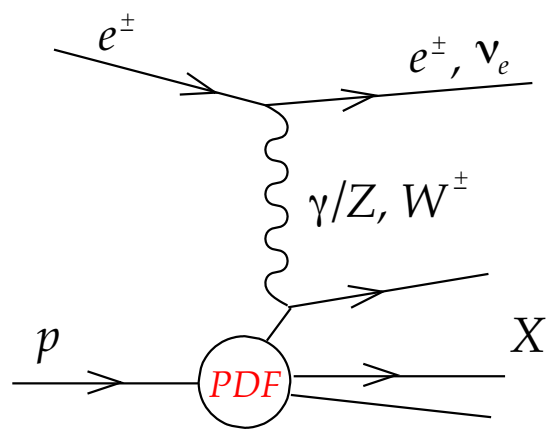

Figure 2: Diagram for neutral NC and charged CC current deep inelastic scattering processes. The symbols denote the particles, the label " $X$ " denotes the hadronic final state.

is scattered off the nucleon via exchange of a $\gamma$ or $Z^{0}$ boson (neutral current, $\mathrm{NC}$, process) or via exchange of a $W^{ \pm}$-boson (charged current, CC). Here, the scattering of an electron (or positron) off the proton is discussed.

The NC (and similarly CC) cross section can be expressed in terms of the generalized structure functions:

$$
\frac{d^{2} \sigma_{N C}^{e^{ \pm} p}}{d x d Q^{2}}=\frac{2 \pi \alpha^{2}}{x Q^{4}}\left[Y_{+} \tilde{F}_{2}^{ \pm} \mp Y_{-} x \tilde{F}_{3}^{ \pm}-y^{2} \tilde{F}_{L}^{ \pm}\right],
$$

where $Y_{ \pm}=1 \pm(1-y)^{2}$ with $y$ being the transferred fraction of the lepton energy. The (generalized) structure function $F_{2}\left(\tilde{F}_{2}\right)$ is the dominant contribution to the cross section, $x \tilde{F}_{3}$ is important at high $Q^{2}$ and $\tilde{F}_{L}$ is sizable only at high $y$. In the framework of perturbative QCD the structure functions are directly related to the parton distribution functions, i.e. in leading order (LO) $F_{2}$ is proportional to the momentum sum of quark and anti-quark distributions, $F_{2} \approx x \sum e_{q}^{2}(q+\bar{q})$, and $x F_{3}$ is related to their difference, $x F_{3} \approx x \sum 2 e_{q} a_{q}(q-\bar{q})$. At higher orders, terms related to the gluon density distribution $\left(\alpha_{S} g\right)$ appear.

In analogy to neutral currents, the inclusive CC ep cross section can be expressed in terms of structure functions, and in LO the $e^{+} p$ and $e^{-} p$ cross sections are sensitive to different quark densities:

$$
\begin{array}{ll}
e^{+}: & \tilde{\sigma}_{C C}^{e^{+} p}=x[\bar{u}+\bar{c}]+(1-y)^{2} x[d+s] \\
e^{-}: & \tilde{\sigma}_{C C}^{e^{-} p}=x[u+c]+(1-y)^{2} x[\bar{d}+\bar{s}] .
\end{array}
$$

At HERA at DESY in Hamburg, electrons (or positrons) were collided with protons at centre-of-mass energies $\sqrt{s}=225-318 \mathrm{GeV}$. The measurements of the $\mathrm{NC}$ and CC cross sections from HERA extend the kinematic regime in $Q^{2}$ by more than two orders of magnitude with respect to the fixed target experiments and cover a wide $x$ range from $10^{-7}$ to 0.7 . At the HERA collider experiments, $\mathrm{H} 1$ and ZEUS, the cross sections of NC and CC DIS are measured with high precision. The measurements of the two experiments are combined [1] accounting for correlations of the systematic uncertainties. The combined cross sections are further used to determine parton distribution functions HERAPDFs.

\subsection{Determination of PDFs}

PDFs are determined from the structure function measurements using the corresponding coefficient functions calculated to a certain order in perturbative QCD (pQCD). The structure functions, and in turn the PDFs, depend on $x$ and $Q$. The $x$-dependence of the parton distributions is not yet calculable in pQCD and has to be parametrized at a certain starting scale $Q_{0}$. The dependence on $Q$ is described by the DGLAP evolution equations [2]. Starting from a parametrization of the nonperturbative PDFs at a starting scale, either by making ad-hoc assumptions on their analytical form or by using the neural-net technology, fits to various sets of experimental data, with HERA DIS data being the backbone, are performed within the DGLAP evolution scheme. The resulting PDFs depend on the order in which the perturbative QCD calculation is performed, the assumptions about the PDF parametrization, the treatment of heavy quarks, the choice for the value of $\alpha_{S}\left(M_{Z}\right)$ and the treatment of the uncertainties. The data sets included in the PDF fit and the consistency of these data sets determines the experimental uncertainty of the PDFs.

\section{HERAPDF}

Starting from the first published HERA PDF set HERAPDF1.0 [1], the parton distributions HERAPDF are determined using combined HERA DIS data, where the correlations of the systematic uncertainties are properly taken into account. This allows the usage of the conventional $\chi^{2}$ tolerance of $\Delta \chi^{2}=1$. Since this QCD 
analysis is solely based on $e p$ data, the PDFs do not depend on the approach for nuclear corrections needed for fixed target data. Several phenomenological schemes of heavy quark treatment can be used in the HERAPDF approach. Therefore direct tests of these schemes are possible.

The full statistics of the HERA inclusive $\mathrm{CC}$ and $\mathrm{NC}$ data are used for NLO and NNLO QCD fits resulting in HERAPDF1.5 [3]. The combined NC and CC cross sections are shown in Fig. 3 and Fig. 4, respectively, together with QCD prediction based on HERAPDF1.5NLO.

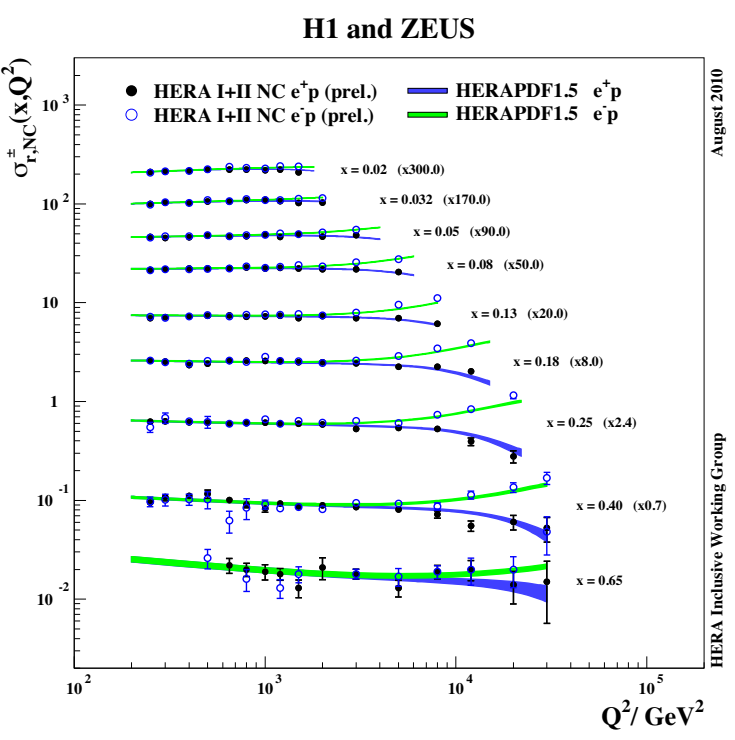

Figure 3: Inclusive DIS cross sections for $N C$ in $e^{ \pm} p$ collisions at HERA. The measurements of the H1 and ZEUS experiments are combined. Open (closed) symbols represent $e^{-} p\left(e^{+} p\right)$ scattering. The shaded curves represent QCD prediction based on HERAPDF1.5NLO.

The QCD analysis HERAPDF1.5 makes use of the same formalism, model and paramatrisation assumptions as used in the HERAPDF1.0. The QCD predictions for the structure functions are obtained by solving the DGLAP evolution equations at NLO (or NNLO) in the $\overline{M S}$ scheme with the renormalisation and factorization scales chosen to be $Q^{2}$. The DGLAP equations yield the PDFs at all values of $Q^{2}$ above the input scale $Q_{0}^{2}$ at which they are parametrized as a functions of $x$. The starting scale $Q_{0}^{2}$ is chosen to be $1.9 \mathrm{GeV}^{2}$ such that the starting scale is below the charm mass threshold. The QCD predictions for the structure functions are obtained by the convolution of the PDFs with the NLO coefficient functions calculated using the general mass

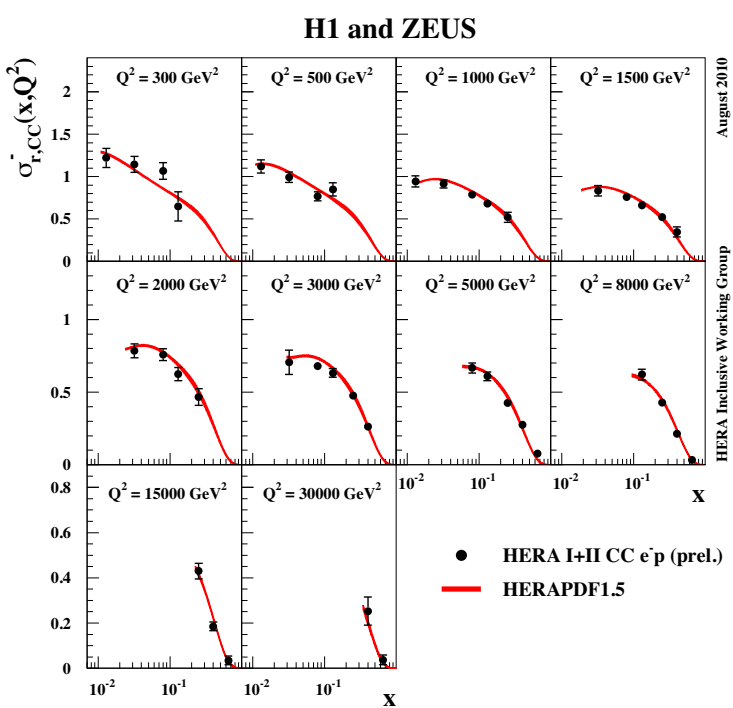

Figure 4: Inclusive DIS cross sections for CC in $e^{-} p$ scattering. The measurements of the $H 1$ and ZEUS experiments are combined. The shaded curves represent $Q C D$ prediction based on HERAPDF1.5NLO.

variable flavour number RT scheme [4].

For the parametrisation of PDFs at the input scale the generic form $x f(x)=A x^{B}(1-x)^{C}\left(1+E x^{2}\right)$ is used. The parametrised PDFs are the gluon distribution $x g$, the valence quark distributions $x u_{v}, x d_{v}$, and the $u$-type and $d$-type anti-quark distributions $x \bar{U}, x \bar{D}$. At the starting scale $Q_{0}^{2}=1.9 \mathrm{GeV}^{2}$ the following relations for quark and antiquark distributions are used: $x \bar{U}=x \bar{u}$ and $x \bar{D}=x \bar{d}+x \bar{s}$. The central fit parametrisation is:

$$
\begin{aligned}
x g(x) & =A_{g} x^{B_{g}}(1-x)^{C_{g}}, \\
x u_{v}(x) & =A_{u_{v}} x^{B_{u_{v}}}(1-x)^{C_{u_{v}}}\left(1+E_{u_{v}} x^{2}\right), \\
x d_{v}(x) & =A_{d_{v}} x^{B_{d_{v}}}(1-x)^{C_{d_{v}}}, \\
x \bar{U}(x) & =A_{\bar{U}} x^{B_{\bar{U}}}\left(1-x^{C_{\bar{U}}}\right), \\
x \bar{D}(x) & =A_{\bar{D}} x^{B_{\bar{D}}}\left(1-x^{C_{\bar{D}}}\right) .
\end{aligned}
$$

The normalisation parameters $A$ are constrained by the quark number and momentum sum-rules, extra constraints for the small- $x$ behaviour of $d-$ and $u$-type quarks are $B_{u_{v}}=B_{d_{v}}, B_{\bar{U}}=B_{\bar{D}}$ and $A_{\bar{U}}=A_{\bar{D}}\left(1-f_{s}\right)$ ( $f_{s}$ is the strange quark fraction), which ensures that $x \bar{u} \rightarrow x \bar{d}$ as $x \rightarrow 0$.

The parton distributions HERAPDF1.5 at NLO at a scale of $Q^{2}=10 \mathrm{GeV}^{2}$ and at NNLO at $Q^{2}=10000$ $\mathrm{GeV}^{2}$ are shown in Fig. 5 and Fig. 6, respectively. In addition to the experimental uncertainties, variation of model inputs and parametrization in the determina- 


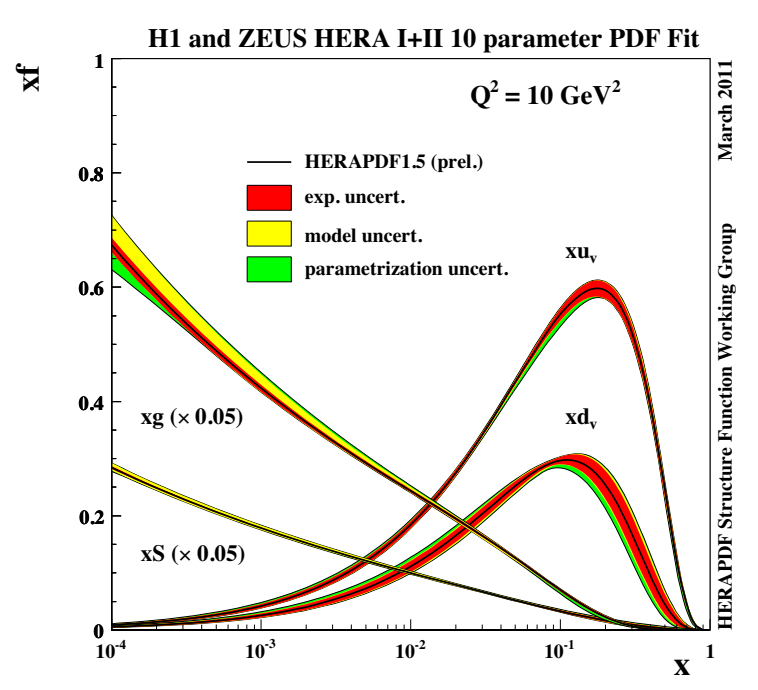

Figure 5: The parton distribution functions from HERAPDF1.5 NLO at $Q^{2}=10 \mathrm{GeV}^{2}$. The gluon and sea distributions are scaled down by a factor of 20. The experimental, model and parametrisation uncertainties are shown.

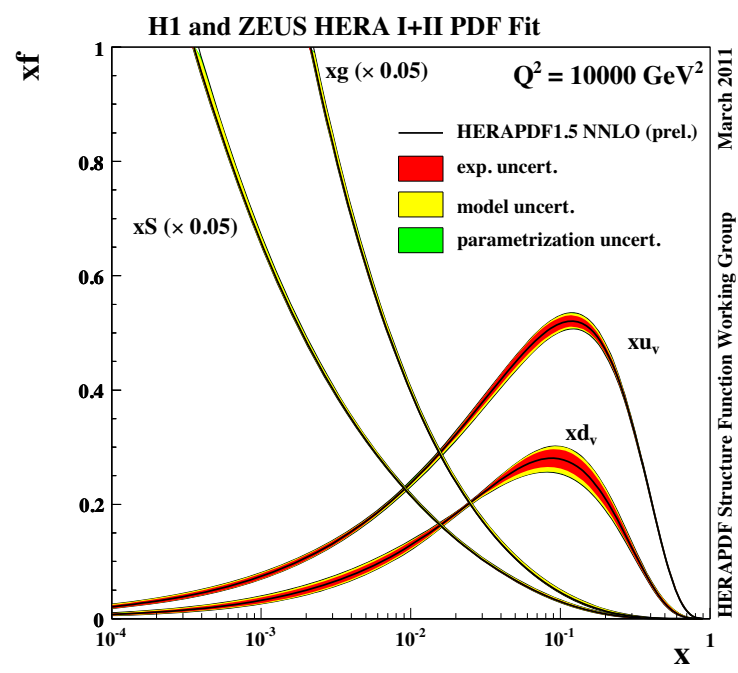

Figure 6: The parton distribution functions from HERAPDF1.5 $N N L O$. The gluon and sea distributions are scaled down by a factor of 20. The experimental, model and parametrisation uncertainties are shown.

tion of HERAPDF are performed and provided as additional eigenvectors. The model uncertainties are evaluated by varying the input assumptions on the minimum $Q^{2}$ of the data used in the fit, $Q_{\min }^{2}$, the strangeness fraction, and the masses of heavy quarks, $m_{c}$ and $m_{b}$. The parametrization uncertainty is formed by an enve- lope of the maximal deviations from the central fit varying parametrization assumptions. HERAPDF1.5NLO and NNLO sets are the recommended HERA PDFs to be used for the predictions of processes at the LHC. The corresponding eigenvectors are available in the LHAPDF library [5].

\section{Benchmarking HERAPDF}

The PDFs are intrinsic properties of the proton and are therefore process-independent. Cross section predictions for processes in proton-(anti)proton collisions can be obtained using HERAPDF, evolved in $Q^{2}$ using the DGLAP equations. In the following, comparisons of the QCD predictions based on HERAPDF1.5 to measurements at Tevatron and the LHC experiments are presented.

The measurements of jet production at hadron colliders is an important instrument for testing the understanding of the PDFs at high $x$, and they also provide additional constraints on the value of $\alpha_{S}\left(M_{Z}\right)$. In Fig. 7 the jet production cross sections as measured by the D0 experiment [6] are presented. The measurements are confronted with the QCD prediction at NLO $[7,8]$ based on HERAPDF1.5NLO. The data are very well described by this prediction.

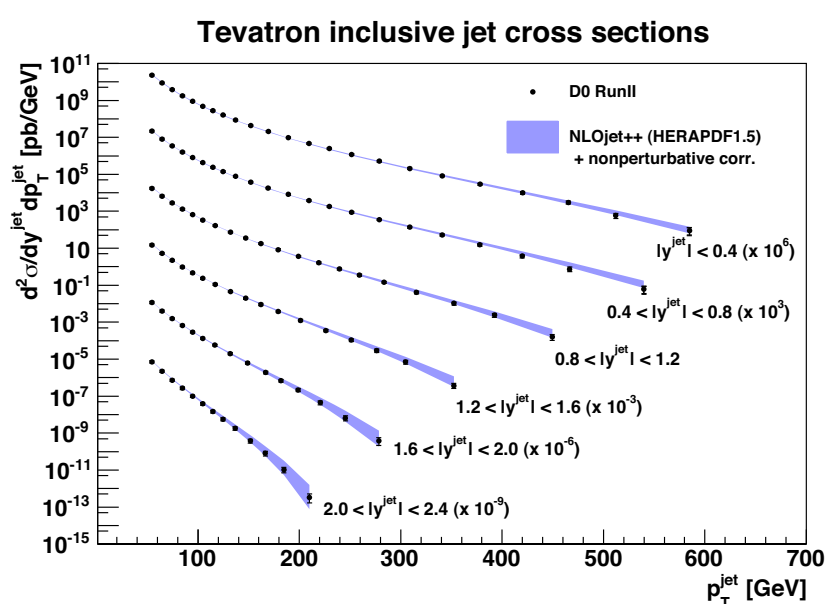

Figure 7: Jet production cross sections as a function of jet transverse momentum for different ranges of pseudorapidity, as measured by the DO collaboration. The data are represented by closed symbols. The measurements are compared to the QCD calculations at NLO based on HERAPDF1.5NLO. The total PDF uncertainty and hadronisation correction uncertainty on the predictions are shown as shaded bands.

Jet production at the LHC is particularly interesting for constraining the parton distribution functions at high 
$x$. In Fig. 8 the jet measurement from the ATLAS experiment [9] in a central rapidity bin is shown in comparison with NLO predictions using HERAPDF1.5NLO and several other PDFs. The QCD prediction using HERAPDF1.5NLO describes the data very well.

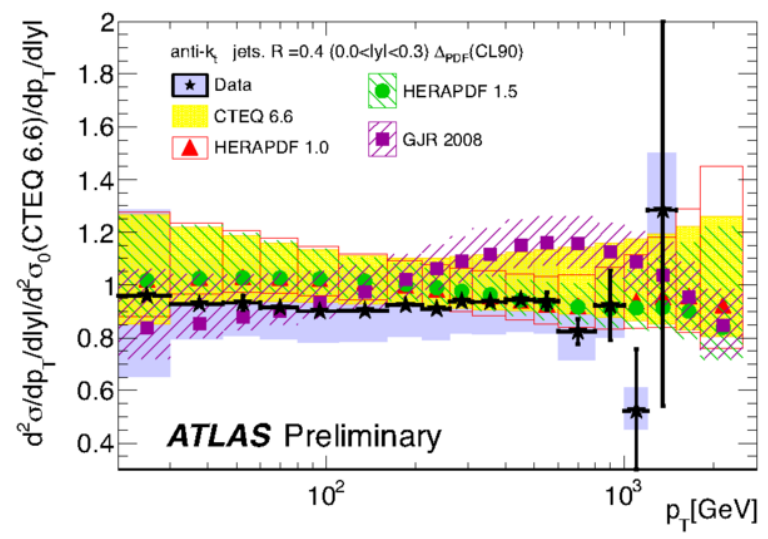

Figure 8: Inclusive jet production cross section as a function of the jet transverse momentum, as measured by the ATLAS collaboration in the rapidity range $0<y<0.3$ The jets are identified using the anti- $k_{t}$ algorithm with $R=0.4$. The data are represented in a ratio (stars) to the $Q C D$ prediction, using CTEQ6.6 [10] as reference PDFs. The central value for the QCD calculation at NLO based on HERAPDF1.5NLO is represented by closed circles surrounded by the error band shown as the hashed area.

Production of electroweak bosons provides important constraints on the light quark distributions. The high precision LHC data can be used not only to compare with theory predictions to differentiate between different PDF sets but also to put constraints on them. For example, the $W$ lepton charge asymmetry $A_{l}(W)$ can help to constrain the $u$ to $d$ quark ratio. This asymmetry can be represented as

$$
A_{l}(W)=\frac{\left(\sigma_{W^{+}}-\sigma_{W^{-}}\right)}{\left(\sigma_{W^{+}}+\sigma_{W^{-}}\right)} \approx \frac{\left(u_{v}-d_{v}\right)}{u_{v}+d_{v}+2 u_{\text {sea }}}
$$

and is sensitive to the valence $u$ and $d$ quark ratio.

The $W$-boson muon asymmetry as measured by the CMS experiment [11] is shown in Fig. 9. The measurement is compared to the NLO predictions [12] obtained using MSTW08 [13], CT10W[14] and HERAPDF1.5 parton densities. The prediction based on HERAPDF1.5NLO describes the data very well.

Top quark pair production at the LHC probes the gluon density at high $x$. In Fig. 10 the cross-section measurement of top pair production $[15,16]$ is shown as a function of the top-quark pole mass in comparison to the approximate NNLO calculations [17] and [18] based on HERAPDF1.5NNLO. The theory uncertainty accounts for the variation of renormalisation and

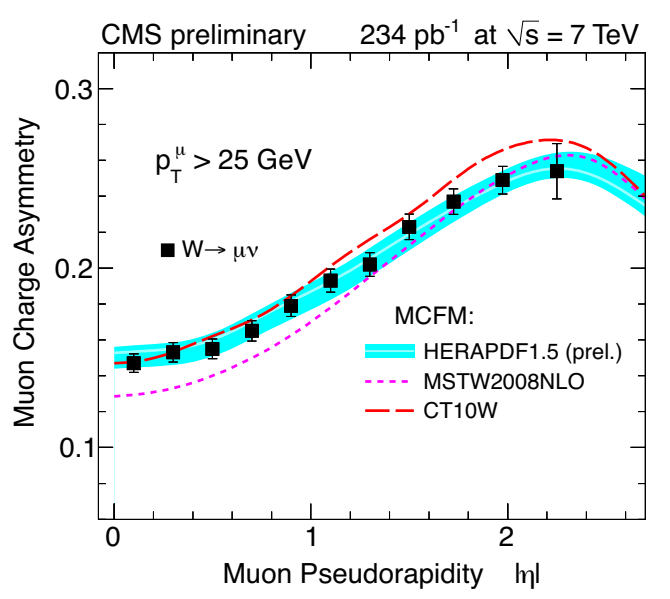

Figure 9: The W-boson muon charge asymmetry as measured by the CMS experiment. The measurement (closed symbols) is compared to the NLO prediction [12] using HERAPDF1.5NLO (shaded band), MSTWOSNLO (dotted line) and CT1OW (dashed line).

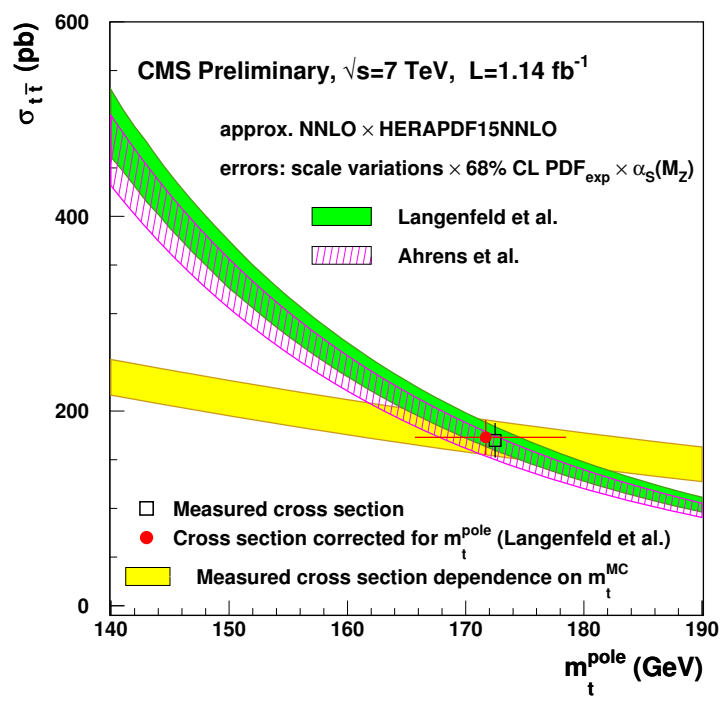

Figure 10: The top-pair production cross section measured by the CMS experiment (open square) shown at the value of the top mass determined in the analysis. The mass dependence of the $t \bar{t}$ cross section according to approximate NNLO QCD predictions [17] and [18] is represented by the shaded and hashed band, respectively. The dependence of the experimental measurement on the assumption on $m_{t}$ in the simulation used for efficiency and detector corrections is shown by the light shaded band. The closed circle represents the cross section measurement, corrected for the top pole mass, extracted using the calculation [17].

factorisation scales, PDFs error and the variation of $\alpha_{S}\left(M_{Z}\right)$ in the PDF. For the PDF uncertainty of HER- 
APDF1.5NNLO, only the eigenvectors for experimental errors are used. The predictions describe the data very well and are used to extract the value of the top mass from the cross section measurement, as described in [16].

Furthermore, the precision of the top pair production measurements at the LHC is essential for studies of the assumptions on parametrization and model parameters in the PDFs. In Fig. 11 the CMS measurement [15] of the $t \bar{t}$ cross section is shown with the prediction [17] using HERAPDF1.5NNLO. In contrast to Fig. 10, also model and parametrization eigenvectors are used in the PDF uncertainty estimation.

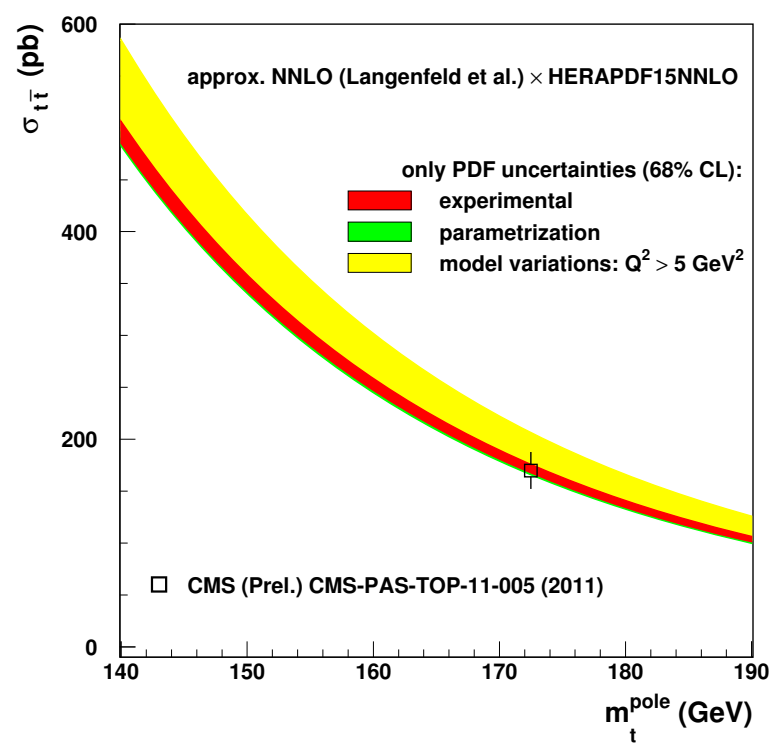

Figure 11: The top-pair production cross section measured by the CMS experiment (open square) shown at the value of the top mass determined in the analysis, compared to the approximate NNLO prediction [17] for the t $\bar{t}$ cross section dependence on the pole mass of the top quark. The shaded band represents the PDF uncertainty of the prediction, broken down into experimental (dark shaded), model (light shaded) and parametrization (covered by the line width) uncertainties.

\section{Global benchmarking excercise}

Presently, the determination of PDFs is carried out by several groups, namely MSTW [19], CTEQ [20], NNPDF [21], HERAPDF [1], AB(K)M [22] and GJR [23]. The large number of PDF parameters and their treatment in the fitting procedure within the different groups results in differences of the PDFs provided. In order to study these differences, a benchmarking exercise is being carried out by the PDF4LHC working group [24] formed by the members of the PDF fitting groups mentioned above. As an example, the NLO prediction for the Higgs cross section $\left(M_{H}=120 \mathrm{GeV}\right)$ at the LHC is shown in Fig. 12 for different PDF sets as a function of $\alpha_{S}\left(M_{Z}\right)$. For the different PDF groups not only the value of $\alpha_{S}\left(M_{Z}\right)$, but also the running of the strong coupling is different, resulting in different cross section predictions.

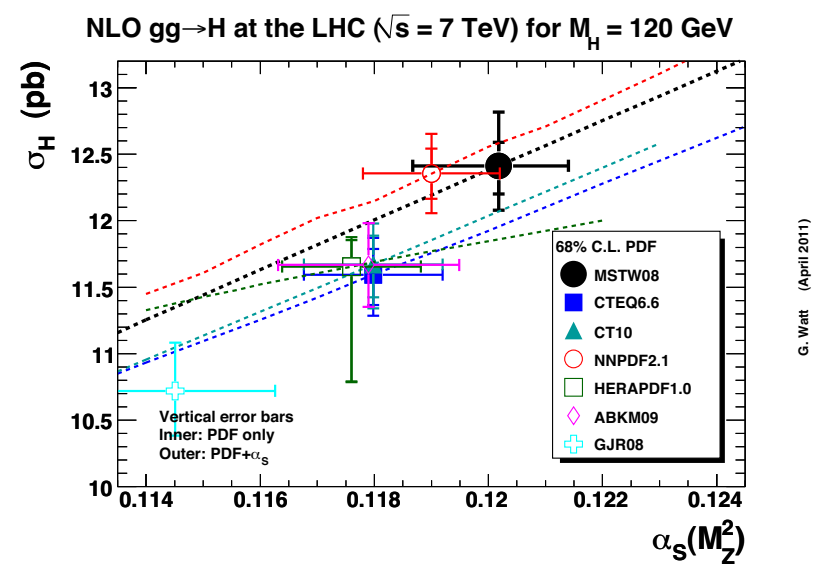

Figure 12: NLO Higgs cross section predictions $\left(M_{H}=120 \mathrm{GeV}\right)$ using different PDFs at the LHC with $\sqrt{s}=7 \mathrm{TeV}$.

The HERAPDF is an active participant in the benchmarking exercise. In contrast to other PDF groups, HERAPDF is not restricted to one particular heavy flavour treatment scheme, several schemes are implemented and can be tested. Also, by providing the PDF eigenvectors for model parameter and parametrization variations, HERAPDF allows for tests of specific parameterisation and model assumptions during the QCD analysis of different data sets. In the following, the inclusion of semi-inclusive DIS data in the QCD analysis of the HERAPDF and the impact of these data on assumptions on $\alpha_{S}\left(M_{Z}\right)$ and the charm quark mass value in the PDF fit is discussed.

\section{Semi-inclusive data in HERAPDFs}

Semi-inclusive measurements in DIS like jet and heavy flavour production, provide additional constraints on the PDFs when included into the QCD analysis together with inclusive DIS data. Jet production is directly sensitive to both the gluon distribution in the proton and the strong coupling $\alpha_{S}$. Therefore, including jet data in the QCD analysis can help disentangling the effects from the gluon and $\alpha_{S}$ in the PDF fit. Similarly, charm and beauty production in $e p$ collisions provide direct 
access to the gluon distribution in the proton, which also depends on the assumption of the charm and beauty mass values used in the PDF fit.

\subsection{Including jet data in the PDF fit: HERAPDF1.6}

In addition to the combined HERA inclusive DIS cross sections as used in the QCD analysis HERAPDF1.5, H1 and ZEUS measurements [25] of jet production cross sections are included in the PDF fit. The resulting parton distributions HERAPDF1.6 [26] are determined using a fixed value of $\alpha_{S}\left(M_{Z}\right)$ and also using $\alpha_{S}\left(M_{Z}\right)$ as a free parameter in the fit. In Fig. 13 the parton distributions for HERAPDF1.6 using a fixed value of $\alpha_{S}\left(M_{Z}\right)$ are compared to those of HERAPDF1.5. Only small changes in the parton distributions, well within the PDF uncertainties, are observed.

H1 and ZEUS HERA I+II PDF Fit with Jets
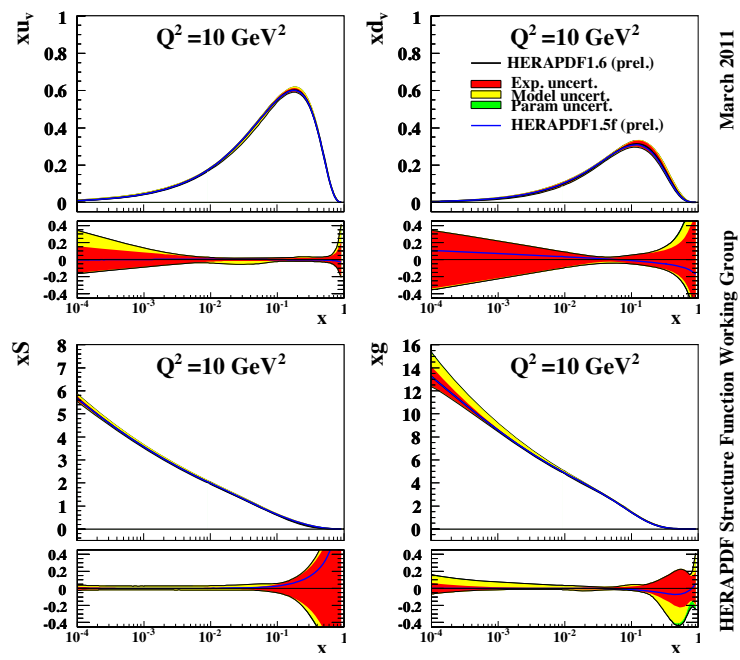

Figure 13: The parton distribution functions from HERAPDF1.6 using a fixed $\alpha_{s}\left(M_{Z}\right)$ compared to those from HERAPDF1.5 at $Q^{2}=$ $10 \mathrm{GeV}^{2}$. The uncertainties of each PDF are presented in the corresponding lower panels.

The impact of the inclusion of jet data in the PDF fit on the gluon distribution and the value of $\alpha_{S}$ is demonstrated in Fig. 14. Here, the PDFs obtained using the inclusive data only (HERAPDF1.5) and the PDFs resulting from including the jet data (HERAPDF1.6) are determined using $\alpha_{S}\left(M_{Z}\right)$ as a free parameter in the QCD analysis. In case of the simultaneous fit of PDFs and $\alpha_{S}$ in HERAPDF1.5, the uncertainties on the gluon PDF becomes large at low $x$ but as soon as the jet data are included, the correlation between the gluon PDF and $\alpha_{S}\left(M_{Z}\right)$ is reduced, resulting in significantly reduced uncertainties on the gluon PDF.
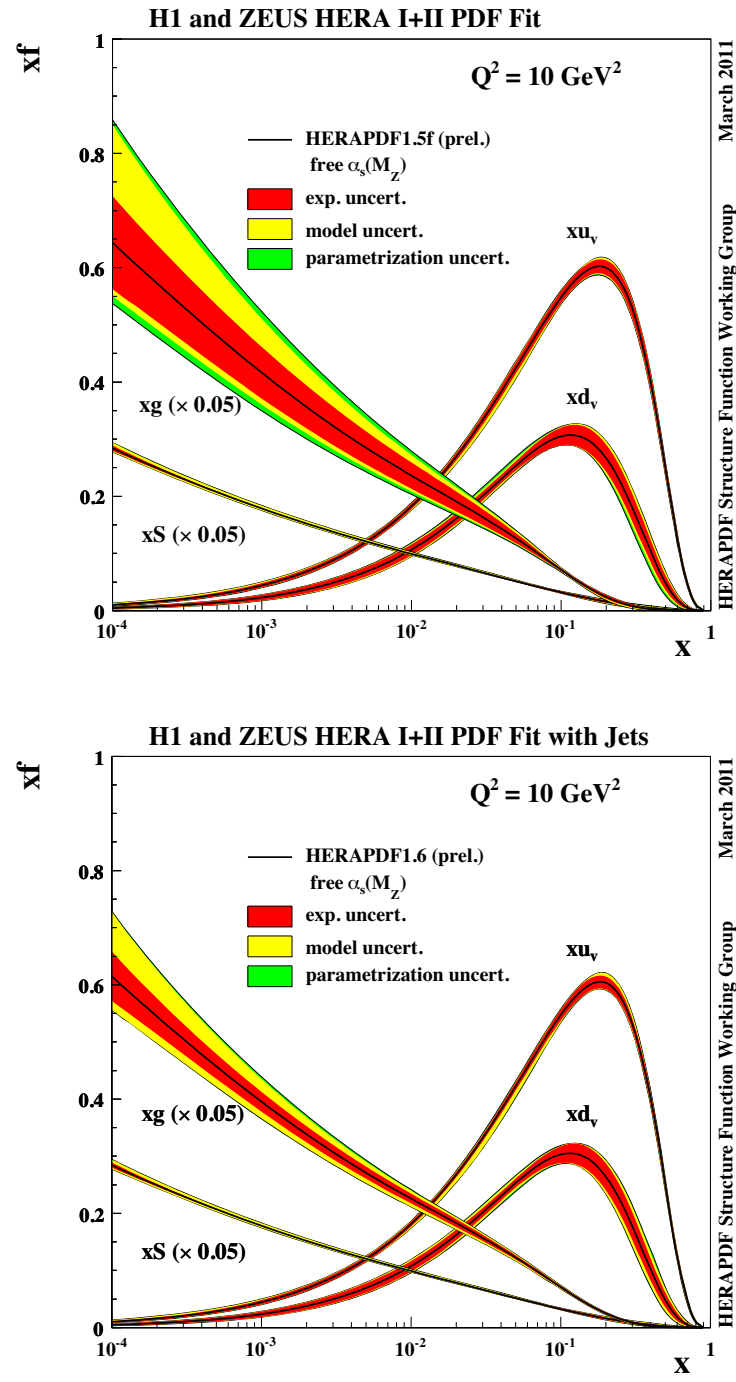

Figure 14: Upper panel: The parton distribution functions from HER$A P D F 1.5$. Lower panel: the parton distribution functions from HERAPDF1.6 (with HERA jet data included in the fit). In both cases, the $Q C D$ analysis is performed treating $\alpha_{s}\left(M_{Z}\right)$ as a free parameter in the fit. The PDFs are presented for $Q^{2}=10 \mathrm{GeV}^{2}$.

In Fig. 15 the quality of the PDF fit in terms of $\chi^{2}$ is represented as a function of the assumption on the value of $\alpha_{S}\left(M_{Z}\right)$. In case of HERAPDF1.5, where only inclusive data are used, a very shallow minimum in the $\chi^{2}$ distribution is observed. The inclusion of the jet measurements in the fit results in a distinct minimum, which allows the simultaneous determination of the PDFs and $\alpha_{S}\left(M_{Z}\right)$. The value of $\alpha_{S}\left(M_{Z}\right)=0.1202 \pm 0.0013(\exp )$ $\pm 0.0007(\mathrm{mod} / \mathrm{param}) \pm 0.0012$ (hadronisation) ${ }_{-0.0036}^{+0.0045}$ (scale) is determined [26]. This result is in very good 


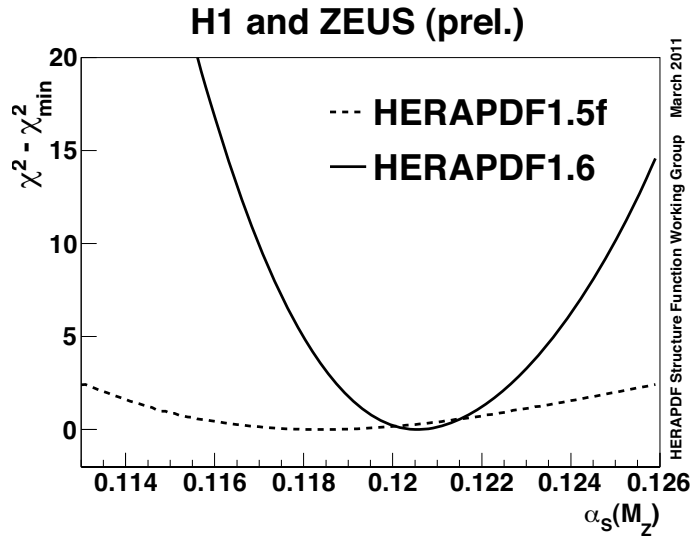

Figure 15: Distribution of $\chi^{2}$ for the PDF fit as a function of the assumption on the $\alpha_{s}\left(M_{Z}\right)$ value. The dashed line corresponds to HERAPDF1.5, where only inclusive DIS data are used. The solid line represents HERAPDF1.6, where jet data from DIS at HERA are included.

\section{H1 and ZEUS (prel.)}

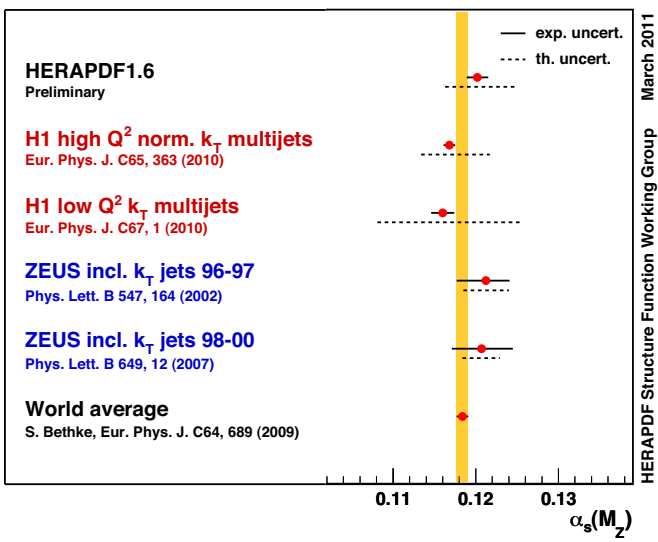

Figure 16: The summary of $\alpha_{s}\left(M_{Z}\right)$ determination results using jet production at HERA as compared to the world average [27]. The upper point corresponds to the simultaneous determination of $\alpha_{s}\left(M_{Z}\right)$ and the PDF, as described in the text. The experimental uncertainty is represented by solid lines, the theory uncertainty is shown by dashed lines.

agreement with different results of $\alpha_{S}$ determination at HERA from jets measurements and the world average as indicated in Fig. 16. It is important to note, that the dominant uncertainty arises from the variation of the renormalization and factorisation scales in the NLO calculation for the jet cross sections. This variation is used to mimic the effect of the missing contribution from the higher orders. Once the NNLO QCD calculations for jet production in $e p$ collisions are provided, a very precise simultaneous determination of $\alpha_{S}\left(M_{Z}\right)$ and PDFs will be achievable using the DIS data.

\subsection{Charm quark measurements in the PDF fit}

The assumption on the number of flavours defines the factorization scheme, used for the PDF determination. The number of flavours in the proton varies depending on the value of the scale, which has to be compared to the threshold, at which charm and beauty quarks can be treated as partons in the proton. This threshold is determined by the mass of charm and beauty quarks. Therefore, the treatment of heavy quarks and the assumptions on their masses have particular importance in the QCD analysis of the proton structure. Different approaches to treat heavy quarks (heavy quark schemes) are used by different PDF fitting groups, corresponding to a different treatment of mass terms in the perturbative calculations, but also implying differences in the interpretation and assumptions on the values of the heavy quark masses. Measurements of charm and beauty production in $e p$ collisions can help to constrain some of these assumptions.

The effect of including the HERA charm data in the QCD PDF fits is studied at HERA. The charm contribution, $F_{2}^{c}$, to the proton structure function $F_{2}$ is measured at $\mathrm{H} 1$ and ZEUS using different charm tagging techniques. These measurements are combined taking into account the correlations of the systematic uncertainties [28] and the result is shown in Fig. 17.

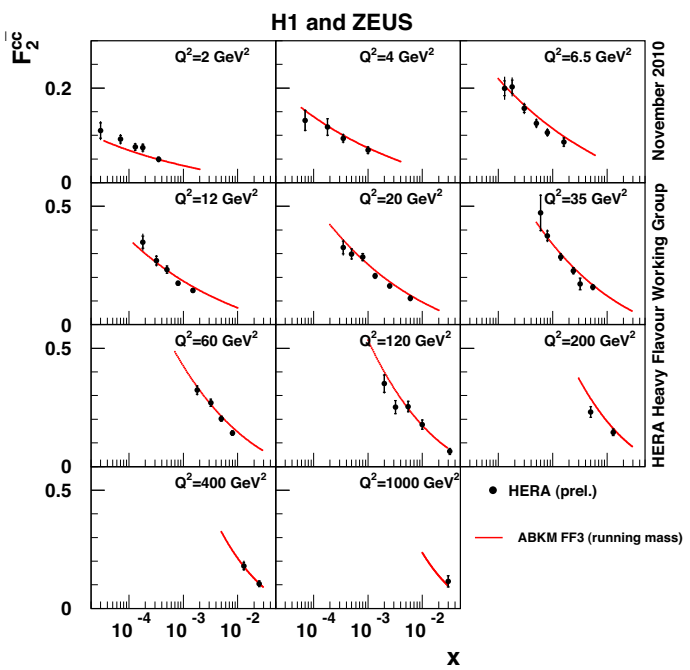

Figure 17: Combined HERA measurement of $F_{2}^{c}$ (closed symbols) as compared to the predictions [29] of the ABKM group.

The combined $F_{2}^{c}$ data are included in the QCD analysis of the inclusive DIS cross sections, and the effect on 
the parton distributions using different assumptions on the charm quark mass, $m_{c}$, is studied [30]. In Fig. 18 the parton distributions HERAPDF1.0 are shown together with the result of the QCD analysis including the combined charm measurements. The best fit quality is obtained using $m_{c}$ closest to the pole mass value [31].

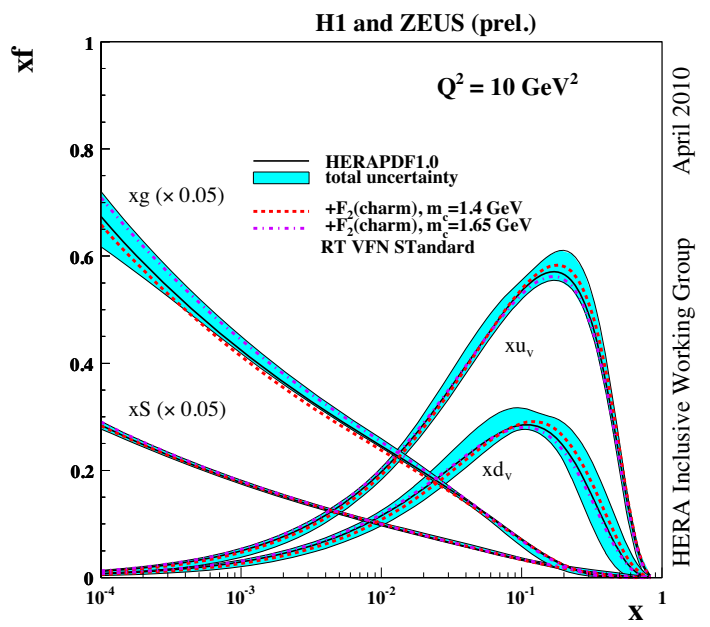

Figure 18: The parton distribution functions from HERAPDF1.0 (shaded bands) at $Q^{2}=10 \mathrm{GeV}^{2}$. The result from the $Q C D$ analysis including combined charm measurements is shown for $m_{c}=1.35 \mathrm{GeV}$ (dashed line) and $m_{c}=1.65 \mathrm{GeV}$ (dash-dotted line).

The sensitivity of the PDF fit to $m_{c}$ when using combined $F_{2}^{c}$ was used to constrain assumptions on $m_{c}$ in different heavy quark schemes [32]. The charm data are used together with different implementations of the variable flavour number schemes (VFNS). The $\chi^{2}$ of the PDF fit including the charm data was determined as a function of the input values of the charm quark mass, $m_{c}^{\text {mod }}$, using different heavy quark schemes. The result is shown in Fig. 19. It is observed that the minimum values of $\chi^{2}$ are comparable for all schemes in spite of the different optimal values of $m_{c}^{\text {mod }}$, corresponding to the $\chi^{2}$ minima.

Although this study is not interpreted as a measurement of the charm quark mass, it provides an important constraint on the assumptions of the $m_{c}$ value by different PDF groups. The important question of a measurement of $m_{c}$ using DIS data is addressed by the ABKM group [29], where the $\overline{M S}$ definition for heavy quark masses in the PDF fit is used. Therefore, the running charm quark mass $m_{c}\left(m_{c}\right)$ can be determined to NLO or NNLO together with the PDF. Important constraints on $m_{c}\left(m_{c}\right)$ from including combined HERA $F_{2}^{c}$ in this analysis are expected.

Different assumptions on $m_{c}^{\text {mod }}$ in VFN schemes im-

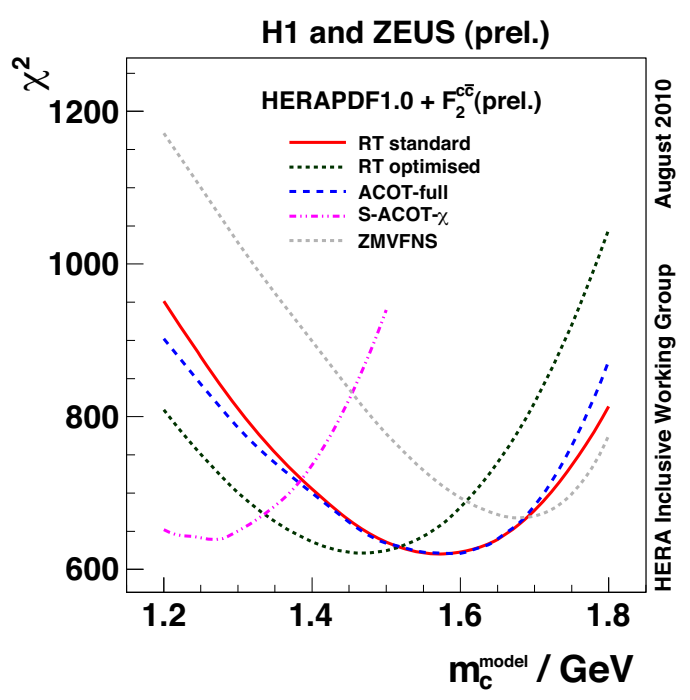

Figure 19: Comparison of the $\chi^{2}$ distributions of fits to the inclusive HERA I $+F_{2}^{c \bar{c}}$ data using different heavy flavour schemes represented as lines of different styles.

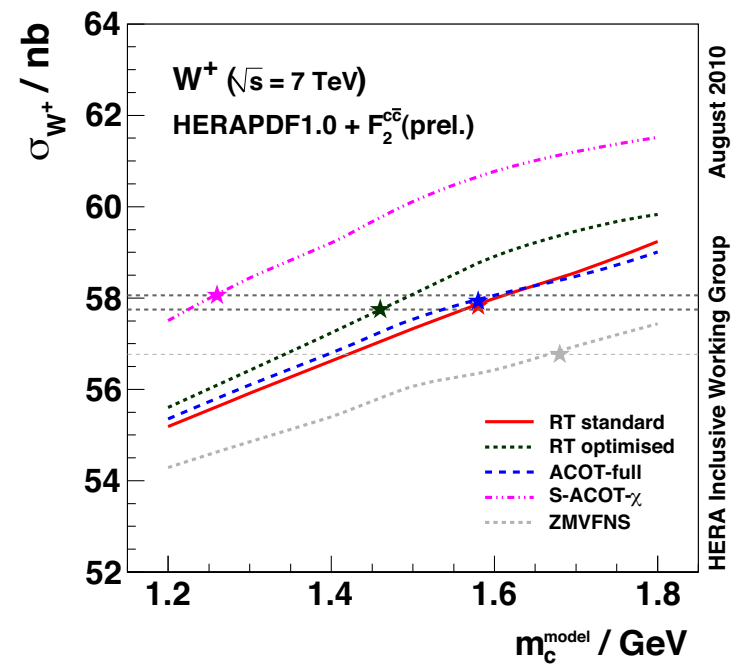

Figure 20: $N L O$ prediction of $\sigma_{W^{+}}$at the LHC for $\sqrt{s}=7 \mathrm{TeV}$ as a function of $m_{c}^{\text {mod }}$ in the input PDF. The lines show predictions for different VFN schemes. The stars show the predictions obtained with the optimal value of $m_{c}^{\text {mod }}$ used in a given scheme. The dashed horizontal lines indicate the range of $\sigma_{W^{+}}$, determined for $m_{c}^{\text {mod }}=m_{c}^{\text {mod }}$ (opt).

pact the charm contribution to the sea quark distribution and thus affect the composition of $x \bar{U}(x)$ from the $x \bar{u}(x)$ and the $x \bar{c}(x)$ contributions. These in turn influence the value of the $W^{ \pm}$and $Z$ cross section predictions at LHC. In Fig. 20 the NLO prediction [12] for the $W^{+}$ 
production cross section is shown, using parton distributions evaluated with different assumptions on $m_{c}^{\bmod }$ in various heavy quark schemes. Taking into account the whole spread of cross section predictions using the studied schemes, an uncertainty of $7 \%$ on the $W^{+}$production cross section arises due to assumption on $m_{c}^{\text {mod }}$ in the PDF. However, when using the values of $m_{c}^{\text {mod }}$ corresponding to the minima from Fig. 19 as constrained by HERA charm data, this uncertainty is reduced to $1 \%$.

\subsection{HERAPDF 1.7}

Finally, a QCD fit is performed which includes all currently available HERA NC and CC inclusive, charm, low energy and jet data (HERAPDF1.7 [33]). In this QCD analysis the studies, performed for including jet and charm data separately, as described above, are repeated. Very consistent results are obtained. This HERAPDF1.7 fit prefers the same $\alpha_{S}$ value as obtained with a fit using only inclusive and jet data (HERAPDF1.6). Similarly, the same optimal $m_{c}$ value is found, as that obtained in a fit when only inclusive and charm data are fitted. A somewhat steeper gluon distribution with respect to the HERAPDF1.6 is obtained, although well within PDF uncertainties, as shown in Fig. 21.

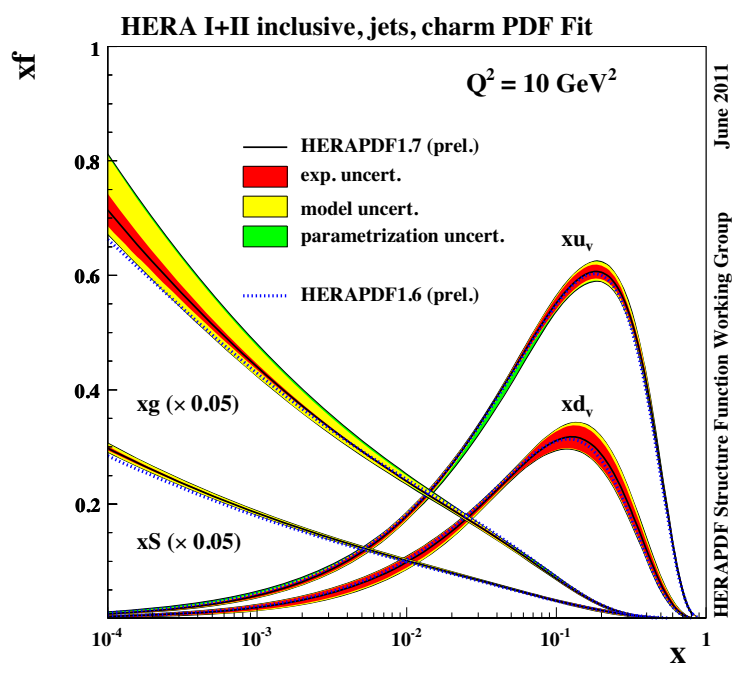

Figure 21: The parton distribution functions from HERAPDF1.7 at $Q^{2}=10 \mathrm{GeV}^{2}$. The gluon and sea distributions are scaled down by a factor of 20. The experimental, model and parametrisation uncertainties are shown separately. For comparison, the central values of HERAPDF 1.6 are also shown.

\section{Summary}

Precision of the parton distribution functions is essential for accurate predictions of cross sections of processes at hadron colliders. The proton PDFs are determined using the experimental data of DIS and protonproton collisions. Combined data of HERA collider experiments provide the most precise constraint on the PDFs at small and medium $x$. HERAPDF is one of the modern QCD analyses in which PDFs are determined. HERAPDFs are based only on the HERA data. The advantages of these PDFs are: no need for nuclear corrections (in contrast to PDFs using the fixed target data), consistent treatment of the systematic uncertainties of the experimental data, and implementation of several phenomenological approaches of the heavy flavour treatment. Currently, HERAPDF1.5 at NLO and NNLO are among the recommended parton densities for predictions of the LHC cross sections. The QCD predictions based on the HERAPDF1.5 describe the measurements at Tevatron and the LHC very well. With increasing precision of the LHC data, particular processes like $W$-boson, jet or top-pair production will provide additional constraints on the PDFs.

Recent developments in the HERAPDF fits include the QCD analyses of HERA inclusive DIS data together with jet and charm measurements. The inclusion of the jet measurements in the HERAPDF analysis reduces the correlation between the gluon distribution and the strong coupling constant. In such a fit, the PDF is determined together with the $\alpha_{S}\left(M_{Z}\right)$ value. The result on $\alpha_{S}\left(M_{Z}\right)$ is in very good agreement with the world average, and the precision is limited by the missing NNLO calculation for jet production. Inclusion of the charm data reduces the correlation between the gluon density and the value of the charm mass used in different schemes of heavy flavour treatment in the PDF fit. In particular, a proper choice of the charm quark mass value is important for accurate QCD predictions of $\mathrm{W}$ and $\mathrm{Z}$ boson production at the LHC. With the proper treatment of the charm mass in the QCD analysis, inclusion of charm measurements should help constraining the running charm quark mass and the PDFs.

\section{References}

[1] F. Aaron et al. [H1 and ZEUS Collaborations], JHEP B 1001, 109 (2010) [arXiv:0911.0884].

[2] V.N. Gribov and L.N. Lipatov, Sov. J. Nucl. Phys. 15, 438 (1972), Sov. J. Nucl. Phys. 15, 675 (1972);

L.N. Lipatov, Sov. J. Nucl. Phys. 20, 94 (1975);

G. Altarelli and G. Parisi, Nucl. Phys. B 126, 298 (1977);

Yu.L. Dokshitzer, Sov. Phys. JETP 46, 641 (1977); 
G. Curci, W. Furmanski, and R. Petronzio, Nucl.Phys. B 175, 27 (1980);

S. Moch, J. Vermaseren, and A. Vogt, Nucl. Phys. B 688, 101 (2004) [arXiv:hep-ph/0403192];

A. Vogt, S. Moch, and J. Vermaseren, Nucl.Phys. B 691, 129 (2004) [arXiv:hep-ph/0404111].

[3] [H1 and ZEUS Collaborations], H1prelim-10-142, ZEUS-prel10-018;

[H1 and ZEUS Collaborations], H1prelim-11-042, ZEUS-prel11-002.

[4] R.S. Thorne and R.G. Roberts, Phys. Rev. D 57, 6871 (1998) [arXiv:hep-ph/9709442].

[5] [LHAPDF], the Les Houches Accord PDF Interface, http://projects.hepforge.org/lhapdf/.

[6] V.M. Abazov et al. [D0 Collaboration], Phys. Rev. Lett. 101, 062001 (2008) [arXiv:hep-ex/0802.2400].

[7] Z. Nagy, Phys. Rev. D 68, 094002 (2003) [arXiv:hep$\mathrm{ph} / 0307268]$.

[8] T. Kluge, K. Rabbertz, and M. Wobisch, [arXiv:hep$\mathrm{ph} / 0609285]$,

http://projects.hepforge.org/fastnlo/.

[9] [ATLAS Collaboration], ATL-PHYS-PUB-2011-005.

[10] P.M. Nadolsky et al., [arXiv:hep-ph/0802.0007].

[11] [CMS Collaboration], CMS-PAS-EWK-11-005.

[12] J. Campbell, K. Ellis, and C. Williams, [MCFM], http://mcfm.fnal.gov/.

[13] A.D. Martin, W.J. Stirling, R.S. Thorne, and G. Watt, Eur. Phys. J. C 63, 189 (2009) [arXiv:0901.0002 [hep-ph]].

[14] H.-L. Lai et al., Phys. Rev. D 82, 074024 (2010) [arXiv:1007.2241 [hep-ph]] .

[15] [CMS Collaboration], CMS Physics Analysis Summary CMSPAS-TOP-11-005 (2011).

[16] [CMS Collaboration], CMS Physics Analysis Summary CMSPAS-TOP-11-008 (2011).

[17] M. Aliev, H. Lacker, U. Langenfeld et al., Comput. Phys. Commun. 182, 1034 (2011) [arXiv:1007.1327].

[18] V. Ahrens et al., JHEP 1009, 097 (2010) [arXiv:1003.5827].

[19] A.D. Martin, W.J. Stirling, R.S. Thorne, and G. Watt, Eur. Phys. J. C 63, 189 (2009) [arXiv:0901.0002].

[20] P.M. Nadolsky et al, Phys. Rev. D 78, 013004 (2008) [arXiv:0802.0007].

[21] R.D. Ball et al., Nucl. Phys. B 809, 1 (2009) [arXiv:0808.1231v4], Nucl. Phys. B 838, 136 (2010) [arXiv:1002.4407].

[22] S. Alekhin, J. Blümlein, S. Klein, and S. Moch, Phys. Rev. D 81, 014032 (2010) [arXiv:0908.2766].

[23] M. Glück, P. Jimenez-Delgado, and E. Reya, Eur. Phys. J. C 53, 355 (2008) [arXiv:0709.0614];

M. Glück, P. Jimenez-Delgado, E. Reya, and C. Schuck, Phys. Lett. B 664, 133 (2008) [arXiv:0801.3618].

[24] [PDF4LHC], http://www.hep.ucl.ac.uk/pdf4lhc/.

[25] F.D. Aaron et al. [H1 Collaboration], Eur. Phys. J. C 65, 363 (2010) [arXiv:0904.3870];

F.D. Aaron et al. [H1 Collaboration], Eur. Phys. J. C 67, 1 (2010) [arXiv:0911.5678];

S. Chekanov et al. [ZEUS Collaboration], Phys. Lett. B 547, 164 (2002) [DESY-02-112];

S. Chekanov et al. [ZEUS Collaboration], Nucl. Phys. B 765, 1 (2007) [DESY-06-128].

[26] [H1 and ZEUS Collaborations], H1prelim-11-034, ZEUS-prel11-001.

[27] S. Bethke, these proceedings.

[28] [H1 and ZEUS Collaborations], H1prelim-09-171, ZEUS-prel09-015.

[29] S. Alekhin and S.-O. Moch, [arXiv:1011.5790].
[30] [H1 and ZEUS Collaborations], H1prelim-10-045, ZEUS-prel10-009.

[31] K. Nakamura et al. [Particle Data Group], J. Phys. G 37, 075021 (2010).

[32] [H1 and ZEUS Collaborations], H1prelim-10-143, ZEUS-prel10-019.

[33] [H1 and ZEUS Collaborations], H1prelim-11-143, ZEUS-prel11-010. 